\title{
Bioinformatical analysis of gene expression signatures of different glioma subtypes
}

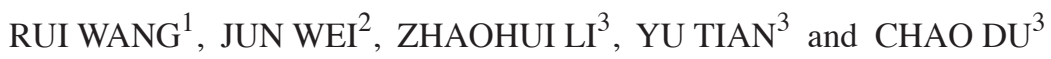 \\ Departments of ${ }^{1}$ Radiology, ${ }^{2}$ Science and Education, and ${ }^{3}$ Neurosurgery, \\ China-Japan Union Hospital of Jilin University, Changchun, Jilin 130033, P.R. China
}

Received January 14, 2016; Accepted July 7, 2017

DOI: $10.3892 / \mathrm{ol} .2017 .7660$

\begin{abstract}
The aim of the present study was to identify the common molecular mechanisms of multiple glioma subtypes, including astrocytoma, glioblastoma and oligodendroglioma, in addition to the specific mechanisms of different types. The gene expression profile set GSE4290 was downloaded from the Gene Expression Omnibus database. Differentially expressed genes (DEGs) from three types of glioma, relative to non-tumor tissue, were calculated by the t-test method with a linear regression model. Kyoto Encyclopedia of Genes and Genomes (KEGG) pathway enrichment analysis of the DEGs was performed. GeneVenn online analysis software was used for the comparison of the DEGs between subtypes. A total of 795 DEGs, including 619 up and 176 downregulated DEGs were screened from the astrocytoma expression profiles; these were enriched in the KEGG pathways of 'neuroactive ligand-receptor interaction' (upregulated) and 'Wnt signaling pathway' (downregulated). Protein-protein interaction networks for astrocytoma, glioblastoma and oligodendroglioma were constructed with 1,617, 7,027 and 1,172 pairs, respectively. A total of 595 common DEGs were obtained between the three subtypes, which were enriched in pathways associated with neural signaling. Glioblastoma is a subtype of astrocytoma; there were 195 DEGs common between these subtypes that were not also associated with oligodendroglioma. DEGs unique to astrocytoma, glioblastoma and oligodendroglioma were associated with the development of the nervous system, the cell cycle and cell matrix components, respectively. The screened DEG p53 gene is likely to be critical for glioma development, including via the Wnt and p53 signaling pathways. Brain-derived neurotrophic factor and cyclin-dependent kinase 1 genes were also likely to be important in the mechanism of glioma development, and were associated with the cell cycle and
\end{abstract}

Correspondence to: Dr Chao Du, Department of Neurosurgery, China-Japan Union Hospital of Jilin University, 126 Xiantai Street, Changchun, Jilin 130033, P.R. China

E-mail: duchao0987@yahoo.com

Key words: glioma, subtypes, target genes, pathways p53 signaling pathways. Immune system-associated and cell matrix component pathways may be unique signaling pathways associated with astrocytoma and oligodendroglioma, respectively.

\section{Introduction}

Glioma is a type of tumor originating in the brain or spine (1). On the basis of histological features, gliomas may be divided into subtypes, including ependymoma, astrocytoma, oligodendroglioma and brainstem glioma (2). Gliomas of the brain typically induce headaches, cranial nerve disorders and seizures, whereas spinal cord gliomas induce pain and numbness in the extremities (3). Depending on the location and cell type of the disease, surgery, radiation therapy and chemotherapy may be combined in glioma treatment (4). However, gliomas are associated with a poor prognosis (5).

The underlying molecular mechanism for glioma tumorigenesis has yet to be established, as it is associated with a number of contributing oncogenes. Therefore, characterizing the molecular mechanisms of the disease is a popular area for research. Previous studies have demonstrated that polymorphisms of DNA repair genes, including excision repair cross-complementing group 1 and 2, and X-ray repair cross-complementing 1, may be associated with an increased risk of glioma development (6). Excessive DNA damage may induce the progression of cancer by causing further mutations that upregulate glioma proliferation (7). In addition, it was previously identified that microRNA-181d regulated the expression of O-6-methylguanine-DNA methyltransferase, potentially inducing glioma progression (8). Although a number of genes and microRNAs associated with glioma have been identified, it is not sufficient to establish a complete strategy for glioma treatment.

Sun et al (9) produced mRNA microarray expression profile data with tumor samples collected from glioma patients (GSE4290), which demonstrated that stem cell factor may be associated with tumor-mediated angiogenesis and the development of glioma. Using bioinformatics analysis of the Sun et al (9) study, Wei et al (10) identified additional differentially expressed genes (DEGs) and the associated transcription factors. The molecular mechanisms of different glioma subtypes were associated with distinct regulatory signaling pathways (10). 
In order to research the common molecular mechanisms of gliomas, in addition to the specific mechanisms of different subtypes, the aforementioned GSE4290 gene expression profile was downloaded and analyzed in the present study. A DEG comparison between different subtypes was performed. This may lay the theoretical foundation for novel strategies of glioma treatment.

\section{Materials and methods}

Data acquisition. The gene expression profile collection GSE4290 (9), which included the expression profile data from 180 samples, was downloaded from the Gene Expression Omnibus (GEO) database (https://www.ncbi. nlm.nih.gov/geo/). The data had been generated using the GPL570 (HG-U133_Plus_2) Affymetrix Human Genome U133 Plus 2.0 microarray platform. The data of 23 samples from the glial cells of epilepsy patients from GSE4290 were used as non-tumor control profiles. The remaining 157 tumor expression profiles included 26 astrocytoma profiles, 50 oligodendroglioma profiles and 81 glioblastoma profiles. The raw data were obtained for the subsequent analysis.

Data preprocessing and DEG screening. The reduced major axis method (11) was used to normalize the raw data with the Affy package (12) in R. Compared with non-tumor expression profiles, the DEGs from each glioma subtype were identified by the T-test method with a linear regression model from the R package limma (13). The threshold for DEGs was $\mid \log \mathrm{FCl}>1.0$ and $\mathrm{P}<0.05$.

Gene ontology $(G O)$ and Kyoto Encyclopedia of Genes and Genomes (KEGG) pathway enrichment analysis of DEGs. The GO database comprises data concerning gene annotations, which primarily includes 3 categories: Molecular function (MF); biological process (BP); and cellular component (CC) (14). KEGG (www.kegg.jp) is a database for the systematic analysis of gene functions. The online tool Database for Annotation, Visualization and Integrated Discovery (DAVID) (15) was used for a KEGG pathway enrichment analysis of the identified DEGs. $\mathrm{P}<0.05$ was considered to indicate a significant enrichment.

Protein-protein interaction (PPI) network construction. STRING is a database of experimentally confirmed and predicted PPIs (16). A PPI network was constructed based on STRING and visualized with Cytoscape 2.8.2 (17) with the threshold of combined score $>0.4$. The degree of connectivity was used to identify hub nodes and remove nodes of low significance.

Module analysis and KEGG enrichment analysis. Modules, i.e., groups of genes with similar functional properties, of the constructed PPI network were identified with ClusterONE (18) in Cytoscape with a threshold of $\mathrm{P}<0.05$. The DEG modules were subsequently used for KEGG pathway enrichment analysis as previously described.

DEG comparison of different subtypes. GeneVenn is an online application for comparing gene lists using Venn diagrams (19).
Table I. Top 10 pathways associated with upregulated and downregulated DEGs in astrocytoma expression profiles.

\begin{tabular}{lrl}
\hline Term & DEGs & P-value \\
\hline $\begin{array}{l}\text { Upregulated pathways } \\
\text { hsa04080: Neuroactive ligand-receptor }\end{array}$ & 29 & $1.57 \times 10^{-9}$ \\
interaction & & \\
hsa04020:Calcium signaling pathway & 22 & $4.84 \times 10^{-8}$ \\
hsa04010:MAPK signaling pathway & 22 & $4.43 \times 10^{-5}$ \\
hsa04540:Gap junction & 12 & $6.55 \times 10^{-5}$ \\
hsa04360:Axon guidance & 14 & $1.23 \times 10^{-4}$ \\
hsa04720:Long-term potentiation & 10 & $1.81 \times 10^{-4}$ \\
hsa04012: ErbB signaling pathway & 11 & $2.61 \times 10^{-4}$ \\
hsa04730:Long-term depression & 8 & $4.56 \times 10^{-3}$ \\
hsa05014:Amyotrophic lateral sclerosis & 6 & $2.13 \times 10^{-2}$ \\
hsa04666:Fc R-mediated phagocytosis & 8 & $2.43 \times 10^{-2}$ \\
Downregulated pathways & & \\
hsa04514:Cell adhesion molecules & 9 & $1.28 \times 10^{-3}$ \\
hsa05222:Small cell lung cancer & 7 & $2.32 \times 10^{-3}$ \\
hsa04610:Complement and coagulation & 6 & $5.09 \times 10^{-3}$ \\
cascades & & \\
hsa04672:Intestinal immune network & 5 & $8.01 \times 10^{-3}$ \\
for IgA production & & \\
hsa04310:Wnt signaling pathway & 8 & $1.11 \times 10^{-2}$ \\
hsa05216:Thyroid cancer & 4 & $1.13 \times 10^{-2}$ \\
hsa05310:Asthma & 4 & $1.13 \times 10^{-2}$ \\
hsa05217:Basal cell carcinoma & 5 & $1.20 \times 10^{-2}$ \\
hsa05020:Prion diseases & 4 & $1.89 \times 10^{-2}$ \\
hsa05330:Allograft rejection & 4 & $2.03 \times 10^{-2}$ \\
\hline a & &
\end{tabular}

DEG, differentially expressed gene.

GeneVenn software was used for comparing DEGs between the glioma subtypes.

\section{Results}

DEG screening and pathway enrichment analysis

Astrocytoma. Compared with non-tumor expression profiles, a total of 863 DEGs, including 624 upregulated and 239 downregulated DEGs, were screened from the astrocytoma expression profile data. The upregulated DEGs were enriched in KEGG pathways including 'neuroactive ligand-receptor interaction', 'calcium signaling pathway', 'MAPK signaling pathway' and 'gap junction', whereas downregulated DEGs were enriched in pathways including 'cell adhesion molecules', 'complement and coagulation cascades' and 'intestinal immune network for IgA production' (Table I).

Glioblastoma. There were 1,520 DEGs, including 969 upregulated and 551 downregulated DEGs, between non-tumor and glioblastoma expression profiles. Upregulated DEGs were enriched in KEGG pathways including "calcium signaling pathway', 'long-term potentiation', 'neuroactive ligand-receptor interaction', 'MAPK signaling pathway' and 'axon guidance', 


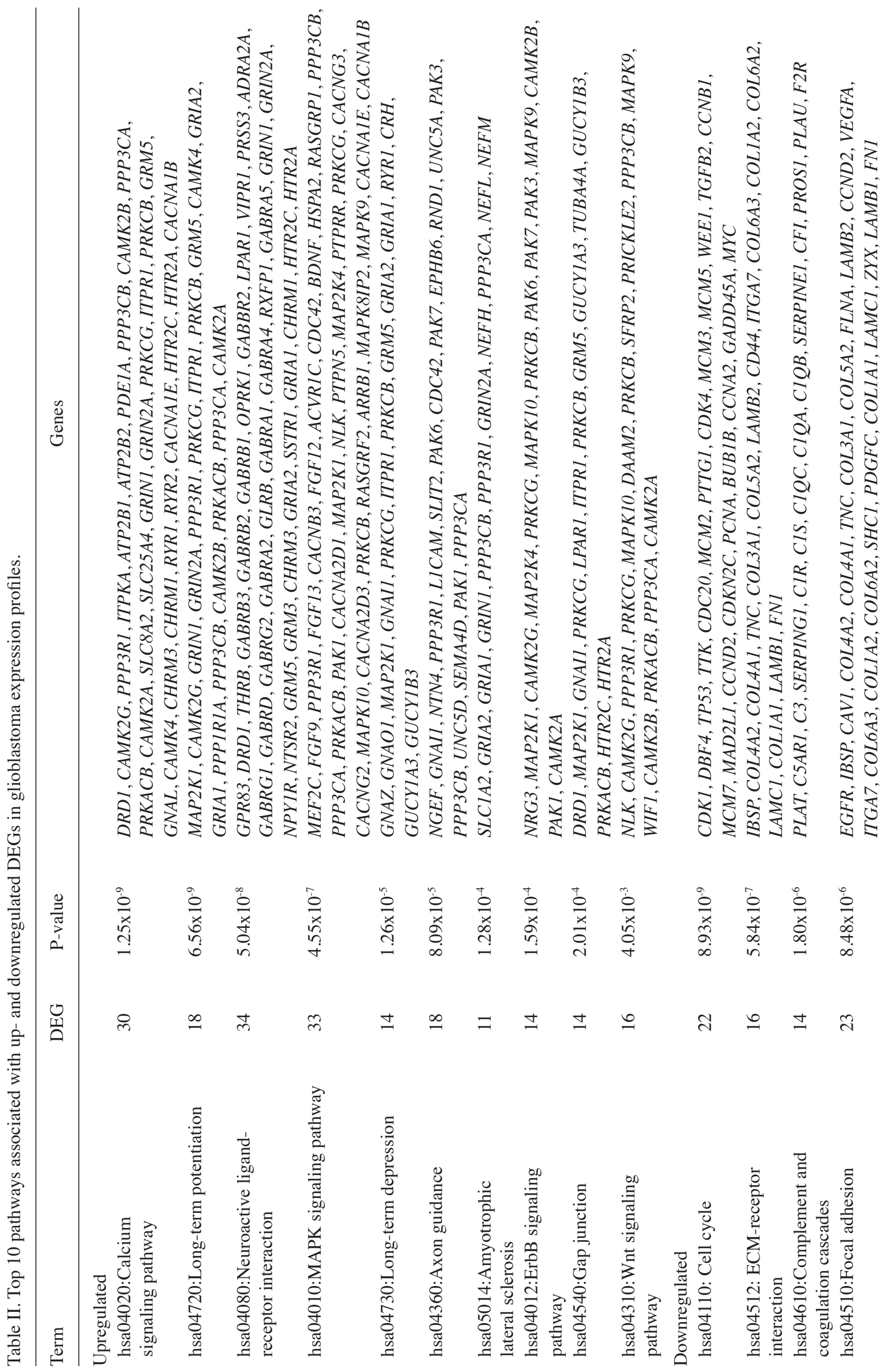


whereas downregulated DEGs were associated with the pathways of 'cell cycle', 'ECM-receptor interaction', 'complement and coagulation cascades', 'focal adhesion' and 'p53 signaling pathway' (Table II).

Oligodendroglioma. Compared with the non-tumor expression profiles, a total of 795 DEGs, including 619 upregulated and 176 downregulated DEGs, were screened from the astrocytoma expression profile data. The upregulated DEGs were enriched in 'neuroactive ligand-receptor interaction', 'calcium signaling pathway', 'axon guidance' and 'gap junction', whereas downregulated DEGs were enriched in 'TGF- $\beta$ signaling pathway', 'p53 signaling pathway' and 'Wnt signaling pathway’ (Table III).

\section{PPI network construction and module analysis}

Astrocytoma. With the threshold of combined score $>0.4$, a PPI network for astrocytoma was constructed with 1,617 pairs. Once nodes with a degree $<2$ were removed, a PPI network for astrocytoma with 506 nodes and 1,590 edges was obtained. In this network, the hub nodes with a degree score $>25$ were $S P Y$, tumor protein p53 (TP53), brain-derived neurotrophic factor $(B D N F), N P Y, S S T, T A C 1$ and SYT1. Module analysis was subsequently performed for this PPI network. Modules A-C were screened, with $\mathrm{P}=2.065 \times 10^{-8}, \mathrm{P}=3.418 \times 10^{-7}$ and $\mathrm{P}=7.808 \times 10^{-4}$, respectively. Module A included 24 nodes and 126 edges; module $\mathrm{B}$ included 21 nodes and 120 edges; module $\mathrm{C}$ included 10 nodes and 31 edges (Fig. 1A). On the basis of the analysis of modules A-C, 8 genes in these modules were enriched in the 'neuroactive ligand-receptor interaction' pathway.

Glioblastoma. A total of 7,027 pairs were identified in the PPI network for glioblastoma. Once nodes with a degree $<2$ were removed, a PPI network with 1,064 nodes and 7,003 edges was obtained. Hub nodes with a degree score $>90$ were cyclin-dependent kinase 1 (CDK1), PCNA, TP53, KNTC1 and $C C N B 1$. A total of 4 modules were screened with $\mathrm{P}<0.05$; modules D-G were screened with $\mathrm{P}<0.001$. Module D included 27 nodes and 178 edges, module $E$ included 27 nodes and 176 edges, module $F$ included 12 nodes and 33 edges (Fig. 1B), and module $\mathrm{G}$ included 7 nodes and 11 edges. Genes in modules $\mathrm{D}-\mathrm{F}$ were enriched in the 'protein processing in endoplasmic reticulum' pathway $\left(\mathrm{P}=1.13 \times 10^{-16}\right)$.

Oligodendroglioma. A total of 1,172 pairs were identified in the PPI network for oligodendroglioma. Once nodes with a degree $<2$ were removed, a PPI network with 419 nodes and 1,040 edges was obtained. SPY, TP53, BDNF, CDC42, SYN1, TAC1, NPY, SYT1, SNAP25, MCM7 and ENO2 were identified as hub nodes, with a degree score $>20$. With the threshold of $\mathrm{P}<0.05$, only module $\mathrm{H}$ was screened. Module $\mathrm{H}$ was associated with $\mathrm{P}<0.001$. Module $\mathrm{H}$ contained 22 nodes and 108 edges (Fig. 1C). The genes in module $\mathrm{H}$ were associated with the pathways of 'neuroactive ligand-receptor interaction' $\left(\mathrm{P}=3.20 \times 10^{-14}\right)$ and 'calcium signaling pathway' $\left(\mathrm{P}=7.75 \times 10^{-10}\right)$.

DEGs comparison of different subtype. As included in Table IV, a total of 595 common DEGs were obtained across all three subtypes of glioma (Fig. 2). The pathways enriched with these 
苮造

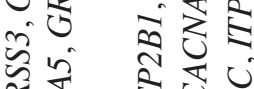

है।

๙

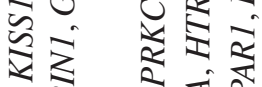

这

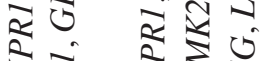

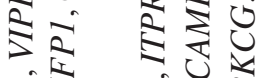

खंख्य

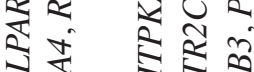

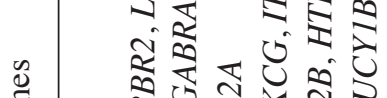

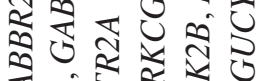

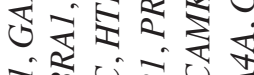

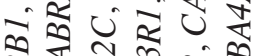

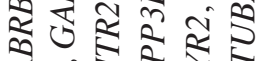

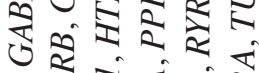

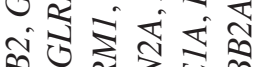

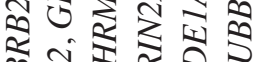

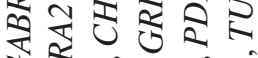

๑

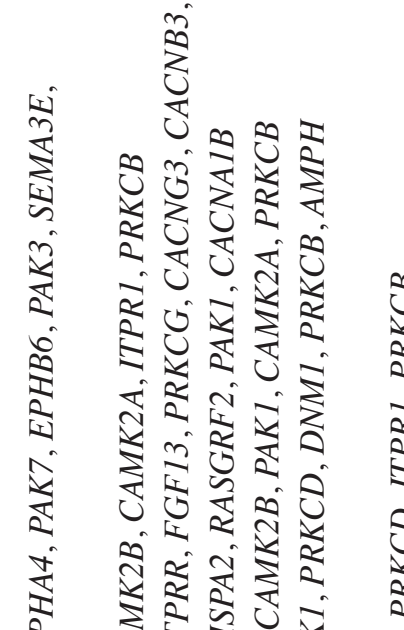

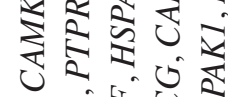

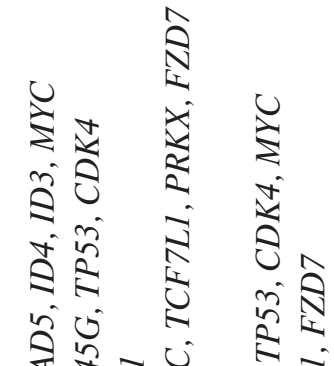

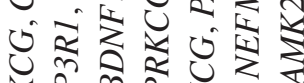

रे बै ह

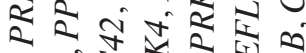

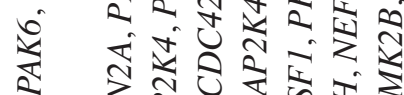

(1)

这

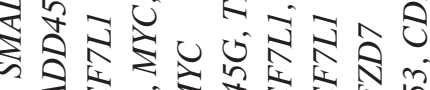
- 娄出

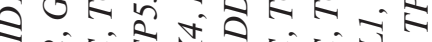
ำ ईิ 4i

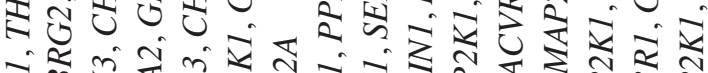

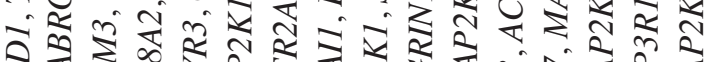

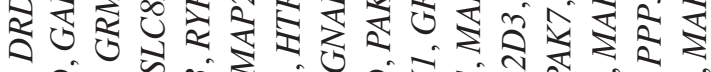
के के फईई

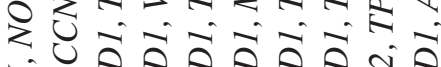

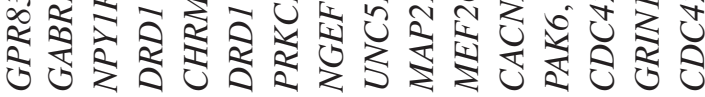

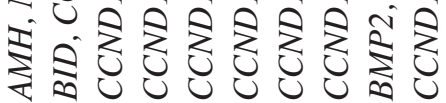

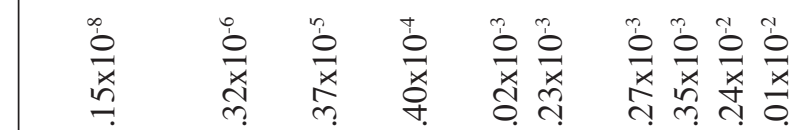

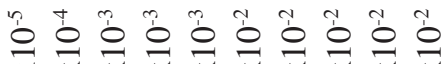
m.

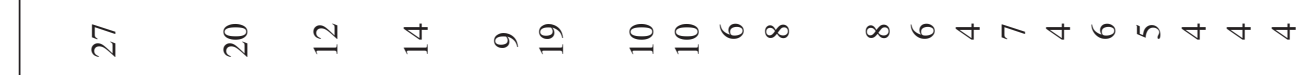

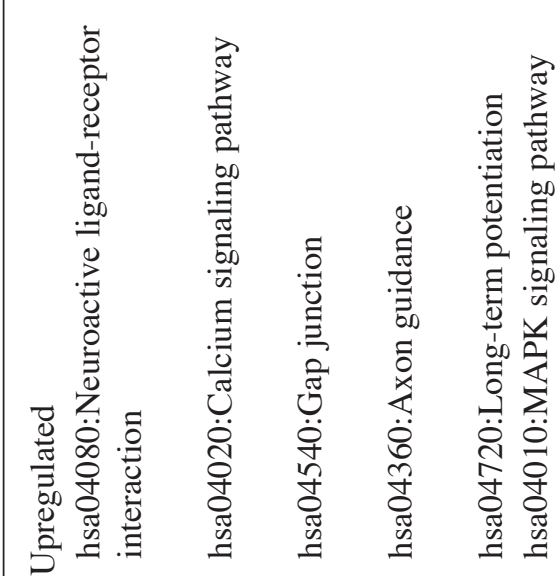

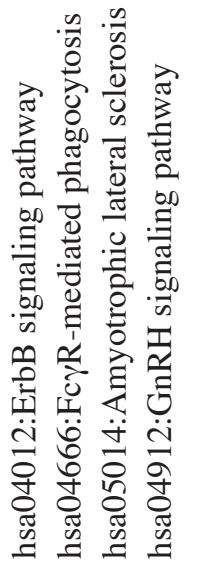

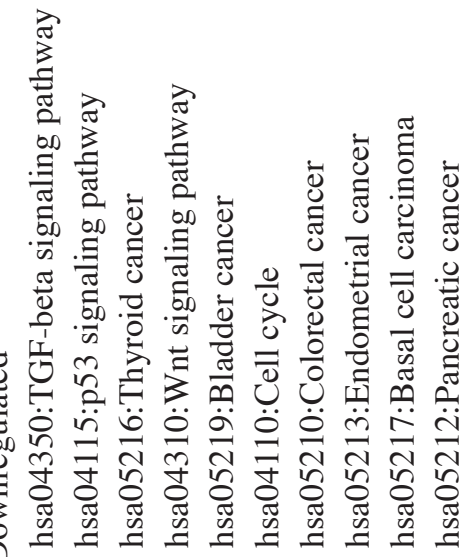


A

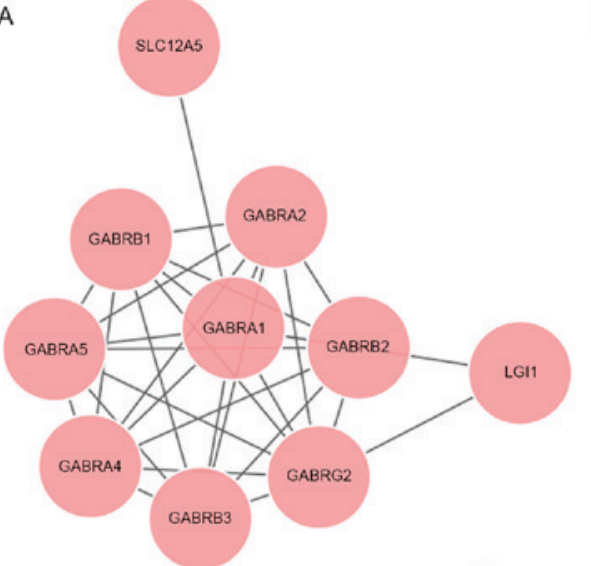

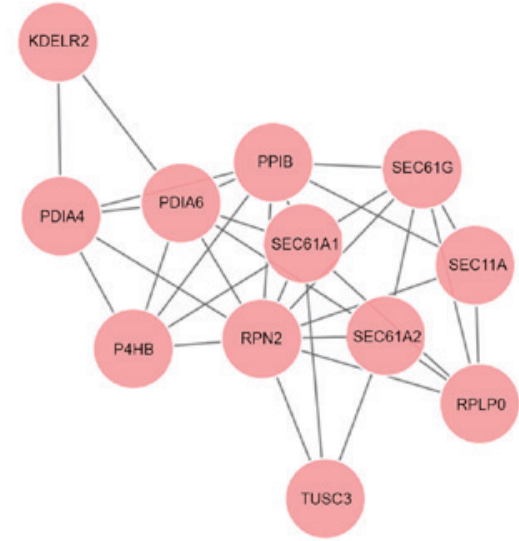

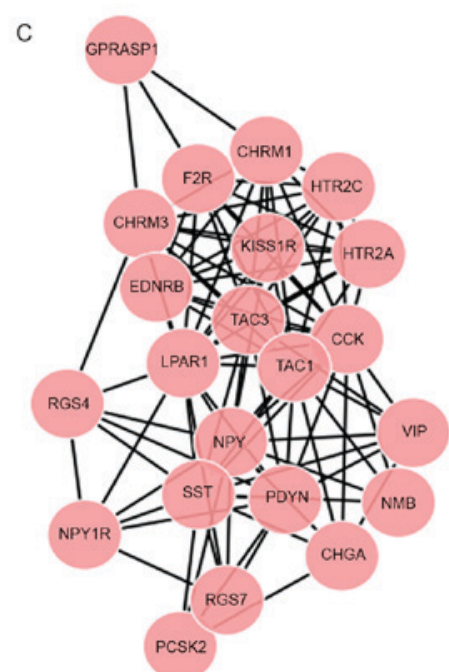

Figure 1. Modules from the PPI network. (A) Module C of the PPI network for astrocytoma; (B) module F of the PPI network for glioblastoma; (C) module H of the PPI network for oligodendroglioma. PPI, protein-protein interaction.

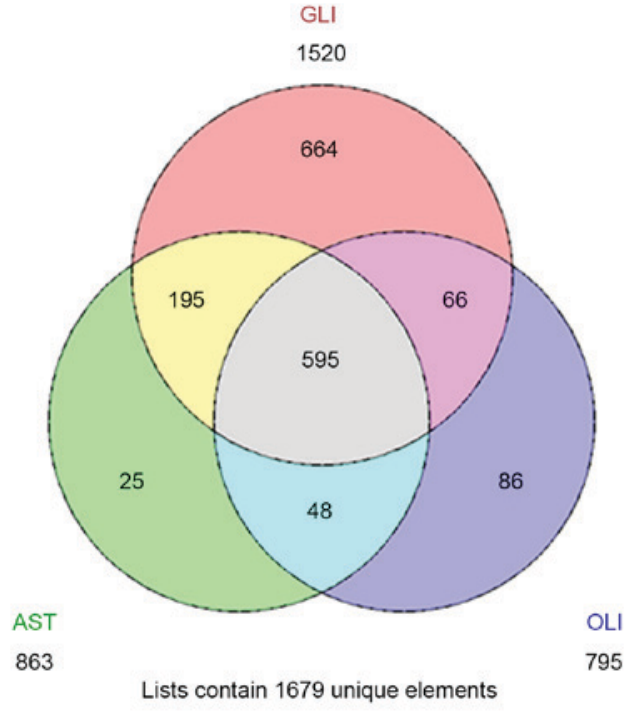

Figure 2. Number of differentially expressed genes that were common between different glioma subtypes. AST, astrocytoma; GLI, glioblastoma; OLI, oligodendroglioma.

genes were associated with neural signaling. Furthermore, glioblastoma is a subtype of astrocytoma; there were 195 common DEGs between the glioblastoma and astrocytoma datasets that were not also associated with oligodendroglioma, which were enriched for immune function-associated pathways. The unique DEGs from astrocytoma, glioblastoma and oligodendroglioma were generally associated with the development of the nervous system, the cell cycle and cell matrix components, respectively (Table IV).

\section{Discussion}

In order to screen for potential therapeutic targets in different glioma subtypes, the GSE4290 profile was downloaded from the GEO for a bioinformatics analysis of the associated molecular mechanisms. In the present study, a total of 595 common DEGs were identified between the three glioma subtypes. The pathways enriched by these genes were associated with neural signaling. There were also a number of unique DEGs and pathways specifically associated with different subtypes.

TP53 was screened as an overlapped DEG between the three glioma subtypes. Additionally, it was enriched in various pathways including the Wnt signaling pathway and the p53 signaling pathway. TP53 is a critical target in the regulation of malignant progenitor cell renewal, differentiation and tumorigenic potential (20). In addition, cellular pathways involving TP53 are frequently dysregulated in glioma tumors (21). Dickkopf-1 was previously demonstrated 
Table IV. GO term enrichment analysis of unique DEGs in three types of glioma.

A, Astrocytoma (enrichment score, 2)

\begin{tabular}{|c|c|c|c|}
\hline GO category & GO term & DEGs & P-value \\
\hline BP & GO:0050767:Regulation of neurogenesis & 3 & $7.66 \times 10^{-3}$ \\
\hline $\mathrm{BP}$ & GO:0051960:Regulation of nervous system development & 3 & $1.01 \times 10^{-2}$ \\
\hline $\mathrm{BP}$ & GO:0060284:Regulation of cell development & 3 & $1.15 \times 10^{-2}$ \\
\hline $\mathrm{BP}$ & GO:0045596:Negative regulation of cell differentiation & 3 & $1.27 \times 10^{-2}$ \\
\hline
\end{tabular}

B, Glioblastoma (enrichment score, 7)

\begin{tabular}{|c|c|c|c|}
\hline GO category & GO term & DEGs & P-value \\
\hline $\mathrm{BP}$ & GO:0022403:Cell cycle phase & 44 & $4.80 \times 10^{-10}$ \\
\hline BP & GO:0000278:Mitotic cell cycle & 40 & $2.09 \times 10^{-9}$ \\
\hline $\mathrm{BP}$ & GO:0022402:Cell cycle process & 50 & $1.26 \times 10^{-8}$ \\
\hline $\mathrm{BP}$ & GO:0000280:Nuclear division & 27 & $1.14 \times 10^{-7}$ \\
\hline $\mathrm{BP}$ & GO:0007067:Mitosis & 27 & $1.14 \times 10^{-7}$ \\
\hline $\mathrm{CC}$ & GO:0005819:Spindle & 22 & $1.29 \times 10^{-7}$ \\
\hline $\mathrm{BP}$ & GO:0000087:M phase of mitotic cell cycle & 27 & $1.64 \times 10^{-7}$ \\
\hline $\mathrm{BP}$ & GO:0048285:Organelle fission & 27 & $2.54 \times 10^{-7}$ \\
\hline $\mathrm{BP}$ & GO:0007049:Cell cycle & 58 & $2.63 \times 10^{-7}$ \\
\hline $\mathrm{BP}$ & GO:0051301:Cell division & 31 & $3.48 \times 10^{-7}$ \\
\hline $\mathrm{BP}$ & GO:0000279:M phase & 33 & $3.88 \times 10^{-7}$ \\
\hline $\mathrm{CC}$ & GO:0015630:Microtubule cytoskeleton & 38 & $3.98 \times 10^{-4}$ \\
\hline
\end{tabular}

C, Oligodendroglioma (enrichment score, 2)

\begin{tabular}{|c|c|c|c|}
\hline GO category & GO term & DEGs & P-value \\
\hline $\mathrm{CC}$ & GO:0044421:Extracellular region part & 12 & $1.40 \times 10^{-3}$ \\
\hline $\mathrm{CC}$ & GO:0005576:Extracellular region & 16 & $1.19 \times 10^{-2}$ \\
\hline $\mathrm{CC}$ & GO:0005615:Extracellular space & 8 & $2.02 \times 10^{-2}$ \\
\hline
\end{tabular}

GO, Gene Ontology; DEG, differentially expressed gene; BP, biological process; CC, cellular component.

to be an inhibitor of the Wnt signaling pathway by inducing TP53 tumor suppression (22). Dysregulation of the TP53 pathway was also necessary for human astrocytoma by regulating the G1-S transition (23). Therefore, alterations to TP53 expression are critical in glioma via the Wnt and p53 signaling pathways.

Compared with non-tumor expression profiles, notable genes, including $B D N F$, were screened from the astrocytoma expression profiles, which were enriched in the KEGG pathways of 'cell adhesion molecules', 'complement and coagulation cascades' and 'Wnt signaling pathway'. BDNF, a member of the nerve growth factor family, is necessary for the survival of striatal neurons in the brain; in human glioma, the expression of $B D N F$ was previously demonstrated to be upregulated and closely associated with pathological grading (24). In addition, Xiong et al (25) identified that mature BDNF could promote the growth of glioma cells in vitro. The expression of $B D N F$ was confirmed to be regulated by the Wnt signaling pathway (25). Therefore, $B D N F$ may be a therapeutic target in astrocytoma.

CDK1 was a hub node of the PPI network for glioblastoma expression profiles. Chen et al (26) identified that the overexpression of $C D K 1$ may have promoted the oncogenesis and progression of glioma, whereas the downregulation of CDK1 inhibited proliferation. Combined with cyclin B1, CDK1 forms a complex that induces the G2-M transition in malignant glioma cells (27). In the present study, $C D K 1$ was associated with the KEGG pathways 'cell cycle' and 'p53 signaling pathway'. For the treatment of human glioblastoma cells, inducing G1 cell cycle arrest, as may be mediated by the p53 pathway, is an effective strategy for suppressing tumorigenicity (28). $C D K 1$ may thus be associated with the mechanisms of glioblastoma by affecting the cell cycle and the p53 signaling pathway.

In the present study, pathways enriched by DEGs common between the three types of glioma were associated 
with neural signaling. The unique genes of astrocytoma and oligodendroglioma were enriched in immune- and cell matrix component-associated pathways, respectively. The simultaneous activation of the Ras and Akt pathways has been demonstrated to induce glioblastoma development in mice (29). Alterations to the immune system were previously observed to be the primary etiology of adult glioma, particularly in the brain (30). In the process of tumor invasion, extracellular matrix proteins, including fibronectin, may also serve an important function in intracerebral invasion (31).

In conclusion, the screened DEG TP53 is likely to be critical for glioma development, including via the Wnt and p53 signaling pathways. $B D N F$ and $C D K 1$ were also possibly important in the mechanism of glioma development, and were associated with the cell cycle and p53 signaling pathways. Immune system-associated and cell matrix component pathways may be unique signaling pathways associated with astrocytoma and oligodendroglioma, respectively. However, further experiments are required to confirm the results of the present study.

\section{References}

1. Hori M,Fukunaga I, Masutani Y, Taoka T, Kamagata K, Suzuki Y and Aoki S: Visualizing non-Gaussian diffusion: Clinical application of q-space imaging and diffusional kurtosis imaging of the brain and spine. Magn Reson Med Sci 11: 221-233, 2012.

2. Chan AS, Leung SY, Wong MP, Yuen ST, Cheung N, Fan YW and Chung LP: Expression of vascular endothelial growth factor and its receptors in the anaplastic progression of astrocytoma, oligodendroglioma, and ependymoma. Am J Surg Pathol 22: 816-826, 1998

3. Pickuth D and Heywang-Köbrunner SH: Neurosarcoidosis: Evaluation with MRI. J Neuroradiol 27: 185-188, 2000.

4. Stupp R, Mason WP, van den Bent MJ, Weller M, Fisher B, Taphoorn MJ, Belanger $\mathrm{K}$, Brandes AA, Marosi C, Bogdahn U, et al: Radiotherapy plus concomitant and adjuvant temozolomide for glioblastoma. N Engl J Med 352: 987-996, 2005.

5. Bruna A, Darken RS, Rojo F, Ocaña A, Peñuelas S, Arias A, Paris R, Tortosa A, Mora J, Baselga J and Seoane J: High TGFbeta-Smad activity confers poor prognosis in glioma patients and promotes cell proliferation depending on the methylation of the PDGF-B gene. Cancer Cell 11: 147-160, 2007.

6. Wrensch M, Kelsey KT, Liu M, Miike R, Moghadassi M, Sison JD, Aldape K, McMillan A, Wiemels J and Wiencke JK: ERCC1 and ERCC2 polymorphisms and adult glioma. Neuro Oncol 7: 495-507, 2005

7. Gurung RL, Lim SN, Khaw AK, Soon JF, Shenoy K, Mohamed Ali S, Jayapal M, Sethu S, Baskar R and Hande MP: Thymoquinone induces telomere shortening, DNA damage and apoptosis in human glioblastoma cells. PLoS One 5: e12124, 2010.

8. Zhang W, Zhang J, Hoadley K, Kushwaha D, Ramakrishnan V, Li S, Kang C, You Y, Jiang C, Song SW, et al: miR-181d: A predictive glioblastoma biomarker that downregulates MGMT expression. Neuro Oncol 14: 712-719, 2012.

9. Sun L, Hui AM, Su Q, Vortmeyer A, Kotliarov Y, Pastorino S, Passaniti A, Menon J, Walling J, Bailey R, et al: Neuronal and glioma-derived stem cell factor induces angiogenesis within the brain. Cancer Cell 9: 287-300, 2006.

10. Wei B, Wang L, Du C, Hu G, Wang L, Jin Y and Kong D: Identification of differentially expressed genes regulated by transcription factors in glioblastomas by bioinformatics analysis. Mol Med Rep 11: 2548-2554, 2015.

11. Bohonak AJ and van der Linde K: RMA: Software for reduced major axis regression, Java version, 2004. http://www.kimvdlinde. $\mathrm{com} /$ professional/rma.html.

12. Gautier L, Cope L, Bolstad BM and Irizarry RA: Affy-analysis of Affymetrix GeneChip data at the probe level. Bioinformatics 20 : $307-315,2004$
13. Ritchie ME, Phipson B, Wu D, Hu Y, Law CW, Shi W and Smyth GK: Limma powers differential expression analyses for RNA-sequencing and microarray studies. Nucleic Acids Res 43: e47, 2015 .

14. Ashburner M, Ball CA, Blake JA, Botstein D, Butler H, Cherry JM, Davis AP, Dolinski K, Dwight SS, Eppig JT, et al: Gene Ontology: Tool for the unification of biology. The gene ontology consortium. Nat Genet 25: 25-29, 2000.

15. Dennis G Jr, Sherman BT, Hosack DA, Yang J, Gao W, Lane HC and Lempicki RA: DAVID: Database for annotation, visualization, and integrated discovery. Genome Biol 4: P3, 2003.

16. Szklarczyk D, Franceschini A, Wyder S, Forslund K, Heller D, Huerta-Cepas J, Simonovic M, Roth A, Santos A, Tsafou KP, et al: STRING v10: Protein-protein interaction networks, integrated over the tree of life. Nucleic Acids Res 43 (Database issue): D447-D452, 2015.

17. Shannon P, Markiel A, Ozier O, Baliga NS, Wang JT, Ramage D, Amin N, Schwikowski B and Ideker T: Cytoscape: A software environment for integrated models of biomolecular interaction networks. Genome Res 13: 2498-2504, 2003.

18. Nepusz T, Yu H and Paccanaro A: Detecting overlapping protein complexes in protein-protein interaction networks. Nat Methods 9: 471-472, 2012.

19. Pirooznia M, Nagarajan V and Deng Y: GeneVenn-A web application for comparing gene lists using Venn diagrams. Bioinformation 1: 420-422, 2007.

20. Zheng H, Ying H, Yan H, Kimmelman AC, Hiller DJ, Chen AJ, Perry SR, Tonon G, Chu GC, Ding Z, et al: p53 and Pten control neural and glioma stem/progenitor cell renewal and differentiation. Nature 455: 1129-1133, 2008.

21. Ishii N, Maier D, Merlo A, Tada M, Sawamura Y, Diserens AC and Van Meir EG: Frequent co-alterations of TP53, p16/CDKN2A, p14 ${ }^{\mathrm{ARF}}$, PTEN tumor suppressor genes in human glioma cell lines. Brain Pathol 9: 469-479, 1999.

22. Wang J, Shou J and Chen X: Dickkopf-1, an inhibitor of the Wnt signaling pathway, is induced by p53. Oncogene 19: 1843-1848, 2000 .

23. Ichimura K, Bolin MB, Goike HM, Schmidt EE, Moshref A and Collins VP: Deregulation of the $14^{\mathrm{ARF}} / \mathrm{MDM} 2 / \mathrm{p} 53$ pathway is a prerequisite for human astrocytic gliomas with G1-S transition control gene abnormalities. Cancer Res 60: 417-424, 2000.

24. Yan Q, Yu HL and Li JT: Study on the expression of BDNF in human gliomas. Sichuan Da Xue Xue Bao Yi Xue Ban 40: 415-417, 2009 (In Chinese).

25. Xiong J, Zhou L, Lim Y, Yang M, Zhu YH, Li ZW, Zhou FH, Xiao ZC and Zhou XF: Mature BDNF promotes the growth of glioma cells in vitro. Oncol Rep 30: 2719-2724, 2013.

26. Chen H, Huang Q, Zhai DZ, Dong J, Wang AD and Lan Q: CDK1 expression and effects of CDK1 silencing on the malignant phenotype of glioma cells. Zhonghua Zhong Liu Za Zhi 29: 484-488, 2007 (In Chinese).

27. Liu WT, Chen C, Lu IC, Kuo SC, Lee KH, Chen TL, Song TS, Lu YL, Gean PW and Hour MJ: MJ-66 induces malignant glioma cells $\mathrm{G} 2 / \mathrm{M}$ phase arrest and mitotic catastrophe through regulation of cyclin B1/Cdk1 complex. Neuropharmacology 86: 219-227, 2014.

28. Medema RH, Kops GJ, Bos JL and Burgering BM: AFX-like Forkhead transcription factors mediate cell-cycle regulation by Ras and PKB through p2 $7^{\mathrm{kip} 1}$. Nature 404: 782-787, 2000.

29. Holland EC, Celestino J, Dai C, Schaefer L, Sawaya RE and Fuller GN: Combined activation of Ras and Akt in neural progenitors induces glioblastoma formation in mice. Nat Genet 25: 55-57, 2000.

30. Rajaraman P, Brenner AV, Butler MA, Wang SS, Pfeiffer RM, Ruder AM, Linet MS, Yeager M, Wang Z, Orr N, et al: Common variation in genes related to innate immunity and risk of adult glioma. Cancer Epidemiol Biomarkers Prev 18: 1651-1658, 2009.

31. Enam SA, Rosenblum ML and Edvardsen K: Role of extracellular matrix in tumor invasion: Migration of glioma cells along fibronectin-positive mesenchymal cell processes. Neurosurgery 42: 599-608, 1998.

This work is licensed under a Creative Commons Attribution-NonCommercial-NoDerivatives 4.0 International (CC BY-NC-ND 4.0) License. 